\title{
Dark and Trigonometric Soliton Solutions in Asymmetrical Nizhnik-Novikov-Veselov Equation with $(2+1)$-dimensional
}

\author{
Haci Mehmet Baskonus* \\ Department of Mathematics and Science Education, Faculty of Education, Harran University, Sanliurfa, \\ Turkey \\ hmbaskonus@gmail.com
}

\section{ARTICLE INFO}

\section{Article History:}

Received 13 February 2019

Accepted 24 July 2019

Available 03 January 2021

Keywords:

(2+1)-dimensional asymmetrical

Nizhnik-Novikov-Veselov equation

Modified exponential function method

Dark and trigonometric solutions

Contour surfaces

AMS Classification 2010:

$35 A x x ; 35 C x x ; 34 M x x$

\section{ABSTRACT}

In this manuscript, new dark and trigonometric function traveling wave soliton solutions to the $(2+1)$-dimensional asymmetrical Nizhnik-Novikov-Veselov equation by using the modified exponential function method are successfully obtained. Along with novel dark structures, trigonometric solutions are also extracted. For deeper investigating of waves propagation on the surface, 2D and 3D graphs along with contour simulations via computational programs such as Wolfram Mathematica, Matlap softwares and so on are presented.

\section{Introduction}

Special functions such as hyperbolic and trigonometric play an important role in nonlinear science arising in physics, applied science, mathematical physics and so on. In this sense, the hyperbolic sine arises in the gravitational potential of a cylinder while the hyperbolic tangent arises in the calculation and rapidity of special relativity [1]. In recent years, many real world problems can be symbolized with the help of special functions. Therefore, scientists investigating properties of special functions need to modify or revise the classical methods [2-45] which are not giving any solutions such problems for explaining more physical meaning of problems. Authors of [54] developed a novel numerical method that possesses the capability of a multi-scale solution of the engineering problems. They showed that their method can solve the non-linear coupled differential equations with high accuracy and precision. A novel multi-resolution method proposed by Seyedi in [55] for solving partial differential equations. He tested this method for the solution of well-known viscous Burger's equation and the obtained results showed superior accuracy in comparison to the finite difference and boundary element methods. Some important models have been investigated by experts in [56-70]. Boiti et al. [46] has introduced a model which is an important applications in incompressible fluids defined as $[47]$

$$
u_{t}+u_{x x x}+3\left(u \int\left(u_{x}\right) d y\right)_{x}=0,
$$

afternamed the $(2+1)$-dimensional asymmetrical Nizhnik-Novikov-Veselov equation (ANNVE). Jian-Guo Liu [48] has derived new Lump-type solutions by using Hirota's bilinear form for Eq.(1). Z.L. Zhao et al have introduced the mixed Lump stripe solutions to the Eq.(1) [49]. M.S. Osman have applied the generalized unified method for finding multi-wave solutions of Eq.(1) with fractional order [50]. This manuscript is organized

*Corresponding Author 
as follows. In section 2 , we present in a detailed manner the modified exponential function method (MEFM). We apply MEFM to the ANNVE to find new dark and trigonometric solutions in section 3 . In the last section of paper, we present a comprehensive conclusion.

\section{General facts of the MEFM}

MEFM is summarized as follows [51-53];

$$
P\left(u, u_{x}, u_{y}, u_{t}, u^{2}, \cdots\right)=0
$$

where $u=u(x, y, t)$, is an unknown function, $P$ is a polynomial in $u(x, y, t)$.

Step 1: Combining the independent variables $x, y$ and $t$ by a dependent variable $\xi$

$$
\begin{gathered}
u(x, y, t)=U(\xi), \xi=k x+w y-c t \\
\frac{\partial u}{\partial x}=k U^{\prime}(\xi), \frac{\partial u}{\partial t}=-c U^{\prime}(\xi),
\end{gathered}
$$

where $k, w, c$ are real constants and non-zero. Putting Eq.(3) into Eq.(2) produces the nonlinear ordinary differential equation (NODE) as following,

$$
N\left(U, U^{\prime}, U^{\prime \prime}, U^{2}, \cdots\right)=0
$$

where $N$ is a polynomial of $U=U(\xi)$.

Step 2: We suppose the solution form of Eq.(5) in the following form;

$$
U(\xi)=\frac{\sum_{i=0}^{N} A_{i}\left(e^{-\Omega(\xi)}\right)^{i}}{\sum_{j=0}^{M} B_{j}\left(e^{-\Omega(\xi)}\right)^{j}},
$$

in which $A_{i} \quad(0 \leq i \leq N)$ and $B_{j} \quad(0 \leq j \leq M)$ are real-constants with $A_{N} \neq 0, \quad B_{M} \neq 0$. Here, $\Omega=\Omega(\xi)$ satisfies the following differential;

$$
\Omega^{\prime}=\mu \exp (\Omega)+\exp (-\Omega)+\lambda,
$$

Eq.(7) is of the following results under the several conditionals defined as;

Family 1 If $\mu \neq 0, \lambda^{2}-4 \mu>0$,

$$
\Omega(\xi)=\ln \left(\frac{-\sqrt{\lambda^{2}-4 \mu}}{2 \mu} \tanh \left(\frac{\sqrt{\lambda^{2}-4 \mu}}{2}\left(\xi+c_{1}\right)\right)-\frac{\lambda}{2 \mu} .\right.
$$

Family 2If $\mu \neq 0, \lambda^{2}-4 \mu<0$,

$$
\Omega(\xi)=\ln \left(\frac{\sqrt{-\lambda^{2}+4 \mu}}{2 \mu} \tan \left(\frac{\sqrt{-\lambda^{2}+4 \mu}}{2}\left(\xi+c_{1}\right)\right)-\frac{\lambda}{2 \mu} .\right.
$$

Family 3 When $\mu=0, \lambda \neq 0, \lambda^{2}-4 \mu>0$,

$$
\Omega(\xi)=-\ln \left(\frac{\lambda}{\exp \left(\lambda\left(\xi+c_{1}\right)-1\right)}\right) .
$$

Family \& Once $\mu \neq 0, \lambda \neq 0, \lambda^{2}-4 \mu=0$,

$$
\Omega(\xi)=\ln \left(-\frac{2 \lambda\left(\xi+c_{1}\right)+4}{\lambda^{2}\left(\xi+c_{1}\right)}\right) .
$$

Family 5 If $\mu=0, \lambda=0, \lambda^{2}-4 \mu=0$,

$$
\Omega(\xi)=\ln \left(\xi+c_{1}\right) .
$$

Step 3: Setting Eqs. $(3,4)$ into the Eq.(2), afterward, we can find the polynomial of $\exp (-\Omega(\xi))$. Considering all the coefficients of the same power of $\exp (-\Omega(\xi))$ to zero gives a system. By solving this system via various computational programs, we can obtain the values of parameters. This process gives many solutions to the model considered.

\section{Implementation of MEFM}

In this section, MEFM has been successfully considered to the ANNVE to find more and novel dark and trigonometric function traveling wave solutions. Our aim is to obtain a new hyperbolic function traveling wave solution by using an expansion method of the Eq.(1). We take the travelling wave transformation as following

$$
u=u(x, y, t)=U(\xi), \quad \xi=k x+w y-c t,
$$

where $k, w, c$ are real constants and non-zero. Substituting Eq.(11) into Eq.(1) along with easily calculations, we find an equation between and as

$$
N=M+2,
$$

Case 1: If we choose $M=1$ and $N=3$, we can write follows;

$$
\begin{aligned}
U & =\frac{A_{0}+A_{1} e^{-\Omega(\xi)}+A_{2} e^{-2 \Omega(\xi)}+A_{3} e^{-3 \Omega(\xi)}}{B_{0}+B_{1} e^{-\Omega(\xi)}} \\
& =\frac{\Upsilon}{\Psi},
\end{aligned}
$$

and

$$
\begin{gathered}
U^{\prime}=\frac{\Upsilon^{\prime} \Psi-\Upsilon \Psi^{\prime}}{\Psi^{2}}, \\
U^{\prime \prime}=\cdots, \\
\vdots
\end{gathered}
$$

where $A_{3} \neq 0, B_{1} \neq 0$. After simple calculation, we can use the following coefficients for new dark and trigonometric function traveling wave soliton solutions as

Case-1.1 If we select following coefficients,

$$
\begin{aligned}
& A_{0}=\frac{-2 k w \lambda B_{0}}{A_{2}+2 k w B_{0}}\left(A_{1}+2 k w \lambda B_{0}\right), \\
& A_{3}=\frac{A_{2}+2 k w B_{0}}{\lambda}, B_{1}=\frac{-A_{2}-2 k w B_{0}}{2 k w \lambda},
\end{aligned}
$$




$$
\begin{gathered}
c=\frac{\lambda k^{3}\left(-4 A_{1}+\lambda\left(A_{2}-6 k w B_{0}\right)\right)}{A_{2}+2 k w B_{0}}, \\
\mu=\frac{\lambda}{A_{2}+2 k w B_{0}}\left(A_{1}+2 k w \lambda B_{0}\right),
\end{gathered}
$$

we have the new dark solution as following under the Family-1 condition,

$$
\begin{aligned}
u_{1}(x, y, t)= & \varpi-\frac{8 \lambda^{2} k w\left(A_{1}+2 k w \lambda B_{0}\right)^{2}}{\left(A_{2}+2 k w B_{0}\right)^{2} f^{2}(x, y, t)} \\
& \times\left(1+\frac{A_{2}+2 k w B_{0}}{2 A_{1}+4 k w B_{0}} f(x, y, t)\right),
\end{aligned}
$$

where $\varpi=\frac{-2 \lambda k w\left(A_{1}+2 k w \lambda B_{0}\right)}{A_{2}+2 k w B_{0}}, f(x, y, t)=$ $-\lambda-\tau \tanh \left(\frac{1}{2} \tau(k x+w y-c t)\right), \quad \tau=$ $\sqrt{\lambda^{2}-\frac{4 \lambda\left(A_{1}+2 k w \lambda B_{0}\right)}{A_{2}+2 k w B_{0}}}$.

For a better understanding of the physical meaning of Eq.(17), 3D and 2D figures along with contour graphs may be seen in Figures (1), (2) and (3) for suitable values of parameters as follows;

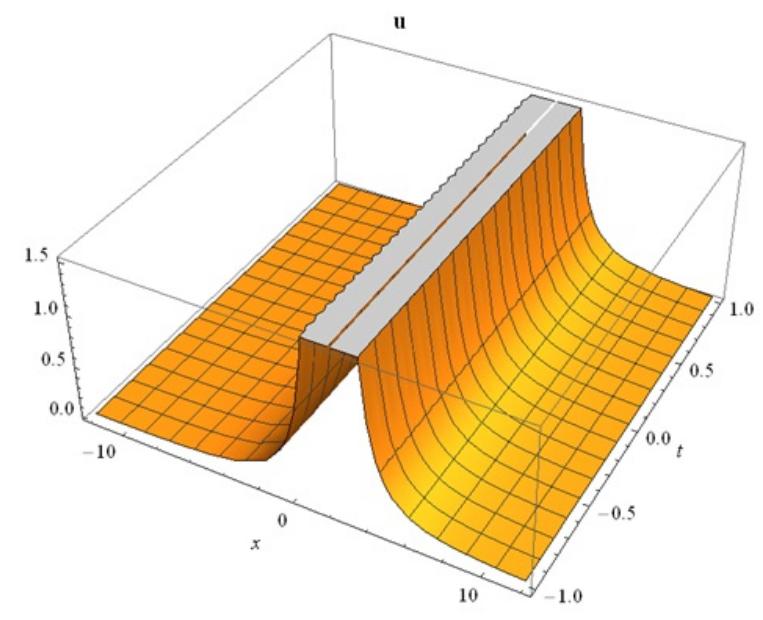

Figure 1. The 3D surface of Eq.(17).

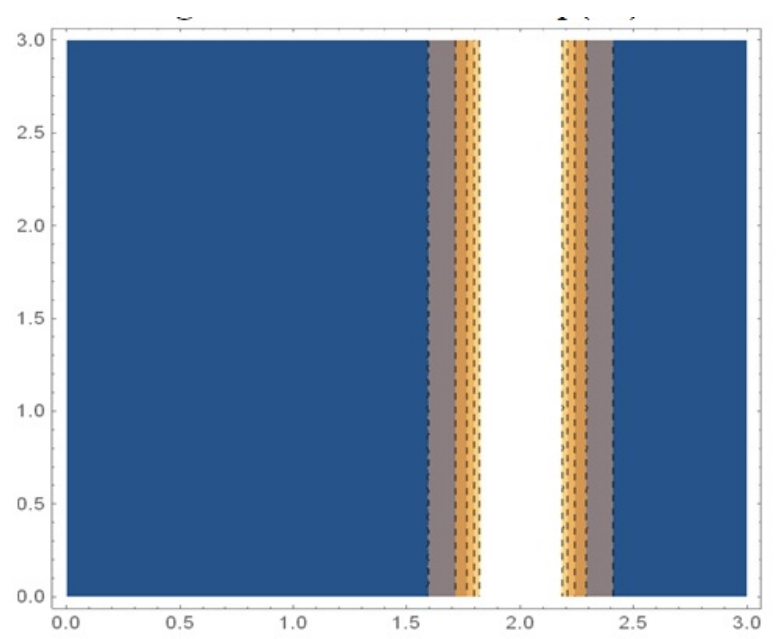

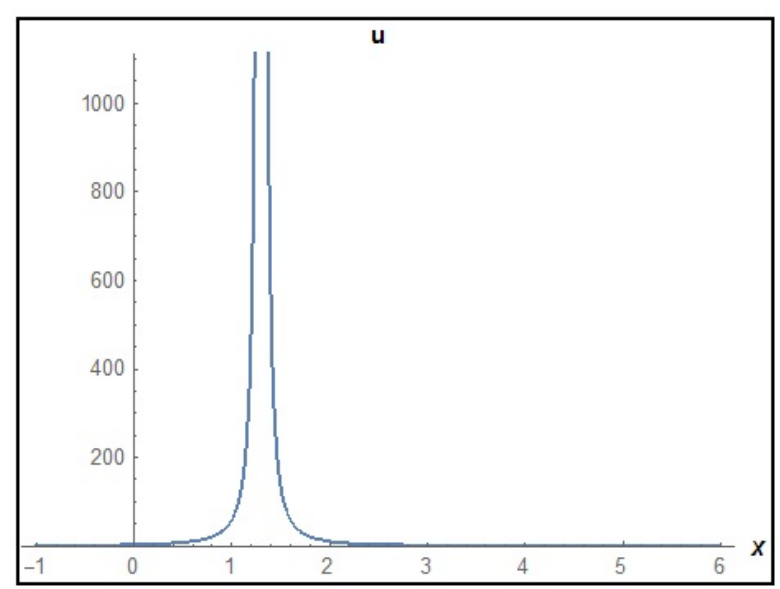

Figure 3. The 2D surface of Eq.(17).

Case-1.2a When

$$
\begin{aligned}
& A_{0}=\frac{B_{0}}{B_{1}^{2}}\left(-\lambda A_{3} B_{0}+A_{1} B_{1}\right), A_{2}=A_{3}\left(\lambda+\frac{B_{0}}{B_{1}}\right), \\
& \mu=\frac{-\lambda^{2}}{2}+\frac{3 A_{1}}{A_{3}}-3 \lambda \frac{B_{0}}{B_{1}}, \\
& c=3 k^{3}\left(\frac{4 A_{1}}{A_{3}}+\lambda\left(-\lambda-\frac{4 B_{0}}{B_{1}}\right)\right), w=\frac{-A_{3}}{2 k B_{1}},
\end{aligned}
$$

we have the new dark solution as following under the Family-1 condition,

$$
\begin{aligned}
u_{2}= & \frac{\kappa \omega+\omega \lambda \tau A_{3} \sqrt{3} B_{1} \tanh \left(\frac{\sqrt{3}}{2} \tau f(x, y, t)\right)}{A_{3} B_{1}^{3}\left(\lambda+\tau \sqrt{3} \tanh ^{2}\left(\frac{\sqrt{3}}{2} \tau f(x, y, t)\right)\right)^{2}} \\
+ & \frac{3 \omega\left(-\lambda A_{3} B_{0}+A_{1} B_{1}\right) \tanh ^{2}\left(\frac{\sqrt{3}}{2} \tau f(x, y, t)\right)}{A_{3} B_{1}^{3}\left(\lambda+\tau \sqrt{3} \tanh ^{2}\left(\frac{\sqrt{3}}{2} \tau f(x, y, t)\right)\right)^{2}},
\end{aligned}
$$

where $\omega=-4 A_{1} B_{1}+\lambda A_{3}\left(4 B_{0}+\lambda B_{1}\right)$, $\kappa=-9 A_{1} B_{1}+\lambda A_{3}\left(9 B_{0}+2 \lambda B_{1}\right), \quad \tau=$ $\sqrt{\frac{-4 A_{1}}{A_{3}}+\lambda\left(\lambda+\frac{4 B_{0}}{B_{1}}\right)}, \quad f(x, y, t)=c_{1}+k x+$ $3 k^{3} \tau^{2} t-\frac{A_{3}}{2 k B_{1}} y$.

$3 \mathrm{D}$ and 2D figures along with contour graphs may be seen in Figures (4),(5) and (6).

Figure 2. The contour surface of Eq.(17). 


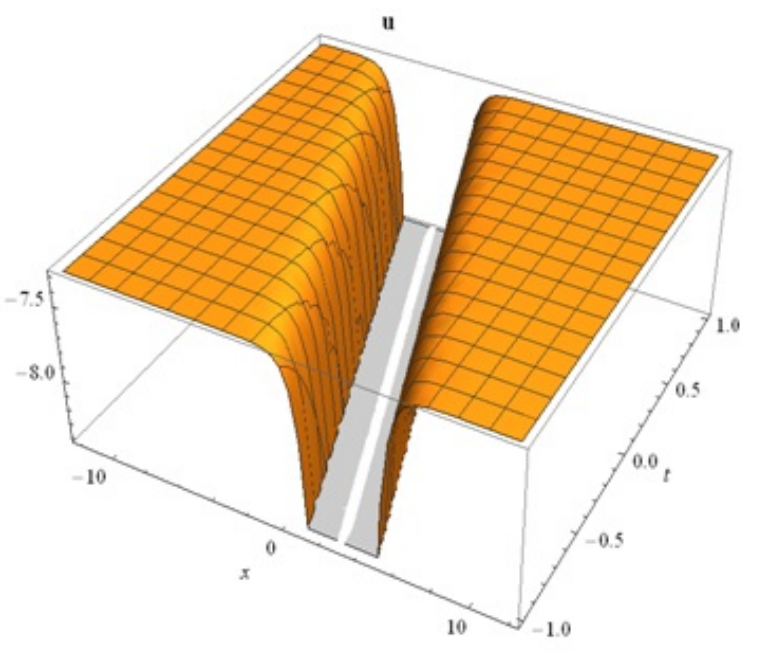

Figure 4. The 3D surface of Eq.(19).

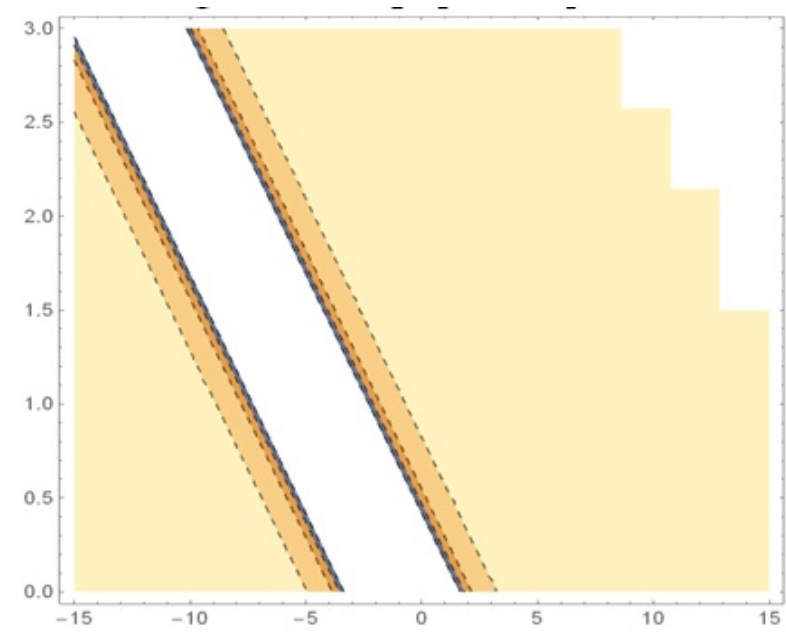

Figure 5. The contour surface of Eq.(19).

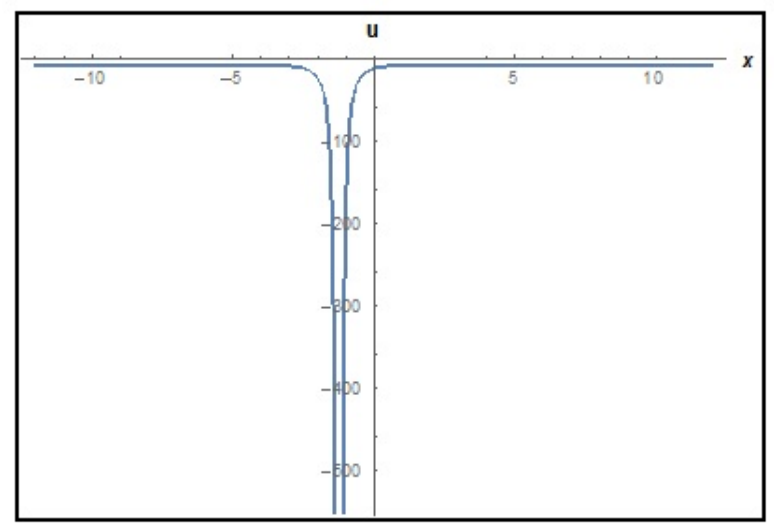

Figure 6. The 2D surface of Eq.(19).

Case-1.2b When we reconsider Eq.(18) under the terms of Family-2 condition, we can find new trigonometric function traveling wave soliton solution

$$
\begin{aligned}
u_{3} & =\frac{\kappa \omega-\omega \lambda \tau \sqrt{3} A_{3} B_{1} \tan \left(\frac{1}{2} \tau f(x, y, t)\right)}{A_{3} B_{1}^{3}\left(\lambda+\tau \sqrt{3} \varpi^{2} \tan ^{2}\left(\frac{1}{2} \tau f(x, y, t)\right)\right)^{2}} \\
+ & \frac{3 \omega\left(\lambda A_{3} B_{0}-A_{1} B_{1}\right) \tan ^{2}\left(\frac{1}{2} \tau f(x, y, t)\right)}{A_{3} B_{1}^{3}\left(\lambda+\tau \sqrt{3} \varpi^{2} \tan ^{2}\left(\frac{1}{2} \tau f(x, y, t)\right)\right)^{2}}
\end{aligned}
$$

in which $\omega=-4 A_{1} B_{1}+\lambda A_{3}\left(4 B_{0}+\lambda B_{1}\right)$, $\kappa=-9 A_{1} B_{1}+\lambda A_{3}\left(9 B_{0}+2 \lambda B_{1}\right), \quad \tau=$ $\sqrt{\frac{12 A_{1}}{A_{3}}+\lambda\left(-\lambda-\frac{4 B_{0}}{B_{1}}\right)}, \varpi=\frac{-4 A_{1}}{A_{3}}+\lambda\left(\lambda+\frac{4 B_{0}}{B_{1}}\right)$, $f(x, y, t)=c_{1}+k x+3 k^{3} \varpi t-\frac{A_{3}}{2 k B_{1}} y$.

$3 \mathrm{D}$ and $2 \mathrm{D}$ figures along with contour graphs may be seen in Figures (7),(8) and (9).

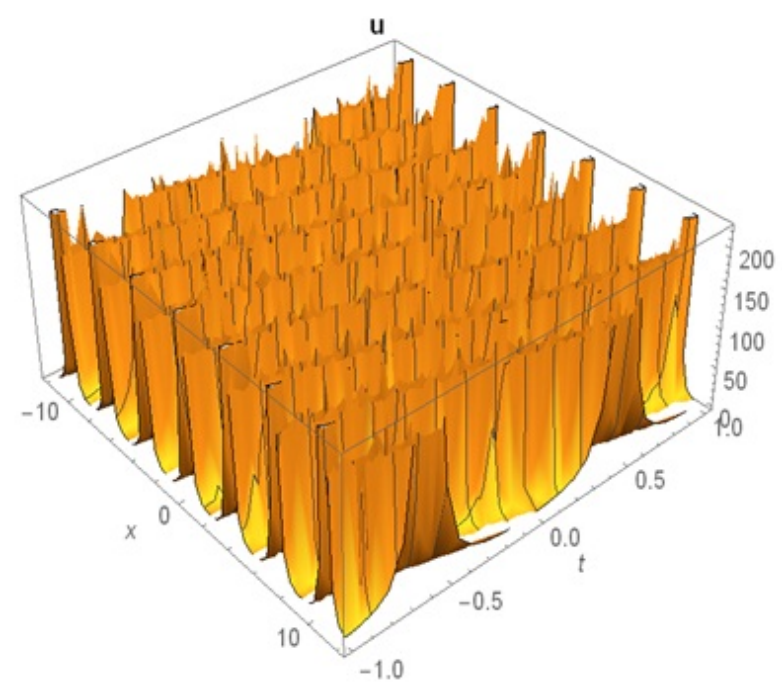

Figure 7. The 3D surface of Eq.(20).

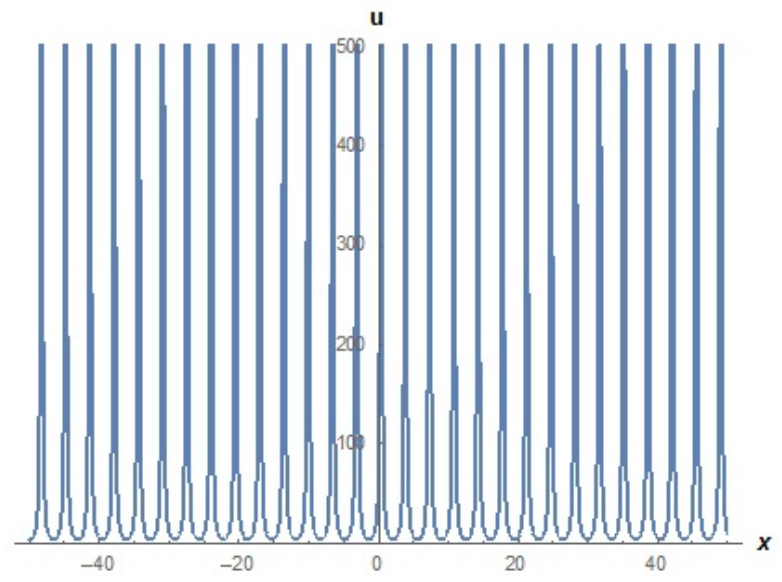

Figure 8. The 2D surface of Eq.(20).

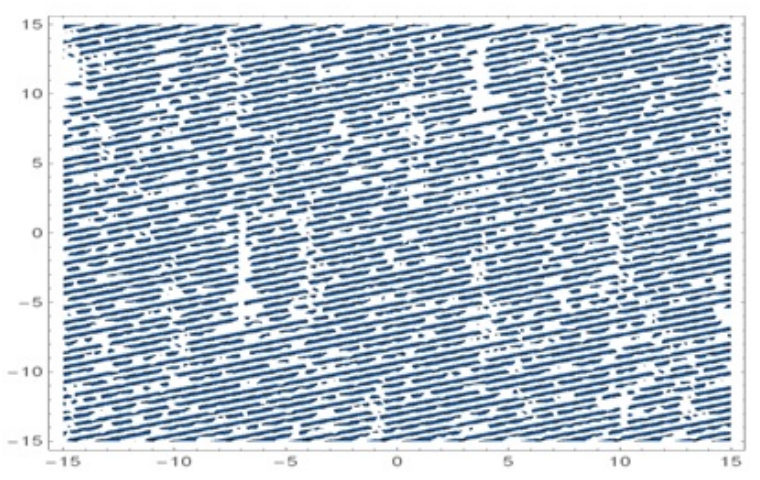

Figure 9. The contour surface of Eq.(20). 


\section{Conclusions}

With the help of MEFM, we have successfully obtained new dark and trigonometric function travelling soliton solutions. For deeper investigating of physical meanings of solutions found in this paper, 2D and 3D graphs along with contour simulations have been plotted. The alternative perspective view of the solutions Eqs. (17, 19, 20) can be viewed from the $3 \mathrm{D}, 2 \mathrm{D}$ graphs along with contour simulations can be also viewed from the Figs. $(1,2,3,4,5,6)$.

Comparing the results produced in this paper with the existing paper in literature, it can be viewed that the results found in this paper are entirely new dark and trigonometric function travelling soliton solutions to the Eq.(1). To the best of our knowledge, the application of MEFM to the $(2+1)$-dimensional asymmetrical NizhnikNovikov-Veselov equation has been not submitted previously. With the help of MEFM, we have successfully obtained new dark and trigonometric function travelling soliton solutions. For deeper investigating of physical meanings of solutions found in this paper, 2D and 3D graphs along with contour simulations have been plotted. The alternative perspective view of the solutions Eqs. $(17,19,20)$ can be viewed from the $3 \mathrm{D}, 2 \mathrm{D}$ graphs along with contour simulations can be also viewed from the Figs. $(1,2,3,4,5,6)$.

Comparing the results produced in this paper with the existing paper in literature, it can be viewed that the results found in this paper are entirely new dark and trigonometric function travelling soliton solutions to the Eq.(1). To the best of our knowledge, the application of MEFM to the $(2+1)$-dimensional asymmetrical NizhnikNovikov-Veselov equation has been not submitted previously.

\section{Acknowledgments}

This study was supported by Scientific Research Projects Unit of Harran University with project number K19015.

\section{References}

[1] Weisstein, E.W. (2002). Concise Encyclopedia of Mathematics, CRC, New York.

[2] Asghar, A., Seadawy, A.R., Lu, D. (2018). New solitary wave solutions of some nonlinear models and their applications. Advances in Difference Equations, 2018(232), 1-12.
[3] Baskonus, H.M., Bulut, H.(2015). Analytical Studies on the $(1+1)$-dimensional Nonlinear Dispersive Modified Benjamin-BonaMahony Equation Defined by Seismic Sea Waves. Waves in Random and Complex Media, 25(4), 1-13.

[4] Aslan, I. (2011). Exact and explicit solutions to nonlinear evolution equations using the division theorem. Applied Mathematics and Computation, 217, 8134-8139.

[5] Araci, S., Ozer, O. (2015). Extended qDedekind-type Daehee- Changhee sums associated with extended q-Euler polynomials. Advance in Difference Equation, 2015(1), 272276.

[6] Cattani, C., Sulaiman, T.A., Baskonus, H.M., Bulut, H. (2018). On the soliton solutions to the Nizhnik-Novikov-Veselov and the Drinfel'd-Sokolov systems. Optical and Quantum Electronics , 50(138), 1-11.

[7] Cattani, C., Sulaiman, T.A., Baskonus, H.M., Bulut, H. (2018). Solitons in an inhomogeneous Murnaghan's rod. European Physical Journal Plus, 133(228), 1-11.

[8] Cattani, C., Ciancio, A. (2016). On the fractal distribution of primes and prime-indexed primes by the binary image analysis. Physica A, 460, 222-229.

[9] Sulaiman, T.A., Bulut, H., Yokus, A., Baskonus, H.M. (2019). On the exact and numerical solutions to the coupled Boussinesq equation arising in ocean engineering. Indian Journal of Physics, 93, 647-656.

[10] Ciancio, A., Baskonus, H. M., Sulaiman, T.A., Bulut, H. (2018). New structural dynamics of isolated waves via the coupled nonlinear Maccari's system with complex structure. Indian Journal of Physics, 92(10), 12811290.

[11] Baskonus, H.M. (2017). New complex and hyperbolic function solutions to the generalized double combined Sinh-CoshGordon equation. AIP Conference Proceeding, 1798(020018), 1-10.

[12] Ciancio, A., Quartarone, A. (2013). A Hybrid Model For Tumor-Immune Competition. U.P.B. Sci. Bull. Series A, 75(4), 125-136.

[13] Arshad, M., Seadawy, A.R., Lu, D. (2017). Bright-dark solitary wave solutions of generalized higher-order nonlinear Schrödinger equation and its applications in optics. Journal of Electromagnetic Waves and Applications, 31(16), 1711-1722.

[14] Baskonus, H.M. (2016). New acoustic wave behaviors to the Davey-Stewartson equation with power-law nonlinearity arising in fluid 
dynamics. Nonlinear Dynamics, 86(1), 177183.

[15] Seadawy, A.R. (2016). Three-dimensional nonlinear modified Zakharov-Kuznetsov equation of ion-acoustic waves in a magnetized plasma. Computers and Mathematics with Applications, 71, 201-206.

[16] Unlukal, C., Senel, M., Senel, B.(2018). Risk Assessment with Failure Mode and Effect Analysis and Gray Relational Analysis Method in Plastic Enjection Prosess. ITM Web of Conferences, 22(01023), 1-10.

[17] Sulaiman, T.A., Yokus, A., Gulluoglu, N., Baskonus, H.M., Bulut, H.(2018). Regarding the Numerical and Stability Analysis of the Sharma-Tosso-Olver Equation. ITM Web of Conferences, 22(01036), 1-9.

[18] Ilhan, O.A., Sulaiman, T.A., Bulut, H., Baskonus, H.M. (2018). On the new wave Solutions to a Nonlinear Model Arising in Plasma Physics. European Physical Journal Plus, 133(27), 1-6.

[19] Ozer, O. (2017). A Note On Structure of Certain Real Quadratic Number Fields. Iranian Journal of Science and Technology, 41(3), 759-769.

[20] Baskonus, H.M., Sulaiman, T.A., Bulut, H., Akturk, T. (2018). Investigations of dark, bright, combined dark-bright optical and other soliton solutions in the complex cubic nonlinear Schrödinger equation with potential. Superlattice and Microstructures, 115, 19-29.

[21] Baskonus, H.M., Askin, M. (2016). Travelling Wave Simulations to the Modified ZakharovKuzentsov Model Arising In Plasma Physics. 6th International Youth Science Forum "LITTERIS ET ARTIBUS", Computer Science and Engineering, Lviv, Ukraine.

[22] Baskonus, H.M., Koc, D.A., Bulut, H. (2016). New travelling wave prototypes to the nonlinear Zakharov-Kuznetsov equation with power law nonlinearity. Nonlinear Science Letters A: Mathematics, Physics and Mechanics, 7(2), 67-76.

[23] Bulut, H., Atas, S.S., Baskonus, H.M. (2016). Some novel exponential function structures to the Cahn-Allen equation. Cogent Physics, 3(1240886), 1-8.

[24] Yokus, A., Sulaiman, T.A., Gulluoglu, M.T., Bulut, H. (2018). Stability Analysis, Numerical and Exact Solutions of the $(1+1)$ Dimensional NDMBBM Equation. ITM Web of Conferences, 22(01064), 1-10.
[25] Yokus, A., Sulaiman, T.A., Baskonus, H.M., Atmaca, S.P. (2018). On the exact and numerical solutions to a nonlinear model arising in mathematical biology. ITM Web of Conferences, 22(01061), 1-10.

[26] Baskonus, H.M., Bulut, H., Emir, D.G. (2015). Regarding New Complex Analytical Solutions for the Nonlinear Partial Vakhnenko-Parkes Differential Equation via Bernoulli Sub-Equation Function Method. Mathematics Letters, 1(1), 1-9.

[27] Ozer, O., Pekin, A. (2015). An Algorithm For Explicit Form of Fundamental Units of Certain Real Quadratic Fields and Period Eight. European Journal Pure and Applied Mathematics, 8(3), 343-356.

[28] Duran, S., Askin, M., Sulaiman, T.A. (2017). New soliton properties to the ill-posed Boussinesq equation arising in nonlinear physical science. International Journal of Optimization and Control, Theories and Applications, $7(3), 240-247$.

[29] Ma, W.X., Li, J., Khalique, C.M. (2018). A Study on Lump Solutions to a Generalized Hirota-Satsuma-Ito Equation in $(2+1)$ Dimensions. Complexity, 2018(9059858), 1-7.

[30] Cilingir, I., Demir, H. (2012). Application of the Hybrid Differential Transform Method to the nonlinear equations. Applied Mathematics, 3(3), 1-10.

[31] Cattani, C.(2018). A review on Harmonic Wavelets and their fractional extension, Journal of Advanced Engineering and Computation, 2(4), 224-238.

[32] Askin, M. (2007). Effect of the Transition Metal Elements on the Relaxation Times in the Agar Solutions. Asian Journal of Chemistry, 19(4), 3191-3196.

[33] Cilingir, I., Demir, H. (2018). New Algorithm for the Lid-driven Cavity Flow Problemwith Boussinesq-Stokes Suspension. Karaelmas Science and Engineering Journal, 8(2), 462-472.

[34] Demir, H. (2014). Temporal differential transform and spatial finite difference methods for unsteady heat conduction equations with anisotropic diffusivity. Gazi University Journal of Science, 27(4), 1063-1076.

[35] Seadawy, A.R.(2017). Ionic acoustic solitary wave solutions of two-dimensional nonlinear Kadomtsev-Petviashvili-Burgers equations in quantum plasma. Mathematical Methods and Applied Sciences, 40, 1598-1607. 
[36] Lu, D., Seadawy, A.R., Khater, M.M.A. (2017). Bifurcations of new multi soliton solutions of the van der Waals normal form for fluidized granular matter via six different methods. Results in Physics, 7, 2028-2035.

[37] Ozer, O. (2018). Determination of Fundamental Units of Real Quadratic Number Fields Related with Specific Continued Fraction Expansions. Egyptian Computer Science Journal, 42(2), 1-12.

[38] Bulut, H., Sulaiman, T.A., Baskonus, H.M., Yazgan, T. (2017). Novel Hyperbolic Behaviors to Some Important Models Arising in Quantum Science. Optical and Quantum Electronics, 49(349), 1-16.

[39] Rosa, M., Gandarias, M.L. (2016). Multiplier method and exact solutions for a density dependent reaction-diffusion equation. Applied Mathematics and Nonlinear Sciences , 1(2), 311-320.

[40] Awati,V.B., Jyoti, M. (2016). Homotopy analysis method for the solution of lubrication of a long porous slider. Applied Mathematics and Nonlinear Sciences , 1(2) (2016) 507-516

[41] Caraballo, T., Herrera-Cobos, M., MarínRubio, P. (2017). An iterative method for non-autonomous nonlocal reaction-diffusion equations. Applied Mathematics and Nonlinear Sciences, 2(1), 73-82.

[42] El-Shaboury, S.M., Ammar, M.K., Yousef, W.M. (2017). Analytical solutions of the relative orbital motion in unperturbed and in Jperturbed elliptic orbits. Applied Mathematics and Nonlinear Sciences, 2(2), 403-414.

[43] Dewasurendra, M., Vajravelu, K. (2018). On the Method of Inverse Mapping for Solutions of Coupled Systems of Nonlinear Differential Equations Arising in Nanofluid Flow Heat and Mass Transfer, Applied Mathematics and Nonlinear Sciences , 3(1), 1-14.

[44] Khalique,C.M., Mhlanga,I.E. (2018). Travelling waves and conservation laws of a $(2+1)$ dimensional coupling system with Kortewegde Vries equation. Applied Mathematics and Nonlinear Sciences , 3(1), 241-254.

[45] Akyildiz, F.T., Vajravelu, K. (2018). Galerkin-Chebyshev Pseudo Spectral Method and a Split Step New Approach for a Class of Two dimensional Semi-linear Parabolic Equations of Second Order. Applied Mathematics and Nonlinear Sciences , 3(1), 255-264.

[46] Boiti, M., Leon, J.J.P., Manna, M., Pempinelli, F. (1985). On the spectral transform of a Korteweg-de Vries equation in two spatial dimensions. Inverse Problems, 2, 271279.
[47] Fan, E. (2009). Quasi-periodic waves and an asymptotic property for the asymmetrical Nizhnik-Novikov-Veselov equation. Journal of Physics A: Mathematical And Theoretical, 42(095206), 1-11.

[48] Liu, J.G. (2019). Lump-type solutions and interaction solutions for the $(2+1)$ dimensional asymmetrical Nizhnik-NovikovVeselov equation. European Physical Journal Plus, 134(56), 1-10.

[49] Zhao, Z.L., Chen, Y., Han, B. (2017). Lump soliton, mixed lump stripe and periodic lump solutions of a $(2+1)$-dimensional asymmetrical Nizhnik Novikov Veselov equation. Modern Physics Letters B, 31, 1750157.

[50] Osman,M.S. (2017). Multiwave solutions of time-fractional (2+1)-dimensional NizhnikNovikov-Veselov equations. Pramana, 88(67), 1-20.

[51] Baskonus, H.M., Bulut, H. (2015). Analytical Studies on the $(1+1)$-dimensional Nonlinear Dispersive Modified Benjamin-BonaMahony Equation Defined by Seismic Sea Waves. Waves in Random and Complex Media, 25(4), 576-586.

[52] Hafez, M.G., Alam, M.N., Akbar, M.A. (2015). Travelling wave solutions for some important coupled nonlinear physical models via the coupled Higgs equation and the Maccari system. Journal of King Saud University Science, 27, 105-112.

[53] Uddin, S., Alam, N., Hossain, S.M.S., Samiu, H., Akbar, M.A. (2014). Some New Exact Traveling Wave Solutions to the (3+1)-Dimensional Zakharov-Kuznetsov Equation and the Burgers Equations via ExpExpansion Method. Frontiers of Mathematics and Its Applications, 1(1), 1-8.

[54] Seyedi, S.H., Saray, B.N., Ramazani, A. (2018). On the multiscale simulation of squeezing nanofluid flow by a highprecision scheme. Powder Technology, 340, 264-273.

[55] Seyedi, S.H. (2018). Multiresolution Solution of Burgers Equation with B-spline Wavelet Basis. arXiv, 1812.10117, 1-20.

[56] Foroutan, M., Manafian, J., Ranjbaran, A. (2018). Lump solution and its interaction to $(3+1)$-D potential-YTSF equation. Nonlinear Dynamics, 92, 2077-2092.

[57] Manafian, J., Lakestani, M. (2019). Lumptype solutions and interaction phenomenon to the bidirectional Sawada-Kotera equation. Pramana, 92(41), 1-15.

[58] Sendi, C.T., Manafian, J., Mobasseri, H., Mirzazadeh, M., Zhou, Q., Bekir, A. (2019). Application of the ITEM for solving three 
nonlinear evolution equations arising in fluid mechanics. Nonlinear Dynamics, 95(1), 669684.

[59] Senel, B. (2020). Fuzzy Dematel analysis on the examination of physical characteristics emergency room affecting the efficiency of doctors. Mathematics in Engineering, Science and Aerospace, 11(1), 77-90.

[60] Zhoua, Y.H., Yang, Y.R., Zhang, H.J. (2018). Stability of non-monotone critical waves in a population dynamics model with spatio-temporal delay. Mathematics in Natural Science, 2, 8-23.

[61] Bulut, H., Kumar, D., Singh, J., Swroop, R., Baskonus, H.M. (2018). Analytic study for a fractional model of HIV infection of CD4+TCD4+T lymphocyte cells. Mathematics in Natural Science, 2(1), 33-43.

[62] Ihan, E., Kiymaz, I.O. (2020). A generalization of truncated M-fractional derivative and applications to fractional differential equations. Applied Mathematics and Nonlinear Sciences, 5(1), 171-188.

[63] Yokus, A., Gulbahar, S. (2019). Numerical Solutions with Linearization Techniques of the Fractional Harry Dym Equation. Applied Mathematics and Nonlinear Sciences, 4(1), 35- 42 .

[64] Singh, J., Kumar, D., Hammouch, Z., Atangana, A. (2018). A fractional epidemiological model for computer viruses pertaining to a new fractional derivative. Applied Mathematics and Computation, 316, 504-515.

[65] Ozer,O. (2020). Fundamental units for real quadratic fields determined by continued fraction conditions. AIMS Mathematics, 5(4), 2899-2908.
[66] Ciancio, A., Quartarone, A. (2013). A hybrid model for tumor-immune competition. U.P.B. Sci. Bull., series A, 75(4), 125-136.

[67] Ciancio, A. (2007). Analysis of time series with wavelets. International Journal of Wavelets, Multire solution and Information Processing, 5(2), 241-256.

[68] Gao, W., Baskonus, H.M., Shi, L. (2020). New investigation of Bats-Hosts-ReservoirPeople coronavirus model and apply to 2019$\mathrm{nCoV}$ system. Advances in Difference Equations, 391, 1-12.

[69] Ciancio, V., Ciancio, A., Farsaci, F. (2008). On general properties of phenomenological and state coefficients for isotropic viscoanelastic media. Physica B: Condensed Matter, 403, 3221-3227.

[70] Durur, H., Ilhan, E., Bulut, H. (2020). Novel Complex Wave Solutions of the (2+1)-Dimensional Hyperbolic Nonlinear Schrödinger Equation. Fractal and Fractional, 4(3), 41.

Haci Mehmet Baskonus received the PhD degree in Mathematics from the Firat University, Turkey, in 2014. He is currently an Associate Professor at the Department of Mathematics and Science Education in Harran University. His research interests include ordinary and partial differential equations, analytical methods for linear and nonlinear differential equations, mathematical physics, numerical solutions of the partial differential equations, fractional differential equations (of course ordinary and partial) and computer programming like Mathematica.

(i) https://orcid.org/0000-0003-4085-3625

An International Journal of Optimization and Control: Theories \& Applications (http://ijocta.balikesir.edu.tr)

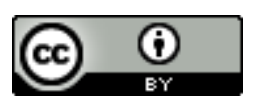

This work is licensed under a Creative Commons Attribution 4.0 International License. The authors retain ownership of the copyright for their article, but they allow anyone to download, reuse, reprint, modify, distribute, and/or copy articles in IJOCTA, so long as the original authors and source are credited. To see the complete license contents, please visit http://creativecommons.org/licenses/by/4.0/. 